\title{
Evaluación de la exposición al monóxido de carbono en vendedores de quioscos. Valencia, Venezuela
}

\author{
Maritza Rojas, ${ }^{1}$ Antonio Dueñas ${ }^{2}$ y Luis Sidorovas $^{1}$
}

RESUMEN Objetivos. Estudiar la intensidad de la exposición al monóxido de carbono (CO), los factores contribuyentes a esta exposición y sus potenciales efectos sobre la salud en vendedores de quioscos ubicados en una avenida de alta densidad de tránsito en Valencia, Venezuela.

Métodos. Se midió la concentración de $\mathrm{CO}$ en el aire (CO-aire) en 16 quioscos y se determinó la concentración sanguínea de carboxihemoglobina ( $\mathrm{COHb}-\mathrm{S}$ ) en los individuos que trabajaban en esos quioscos y en un grupo de control formado por docentes, estudiantes y obreros de la Universidad Simón Rodríguez, ubicada en una zona montañosa, apartada, sin contaminación ambiental aparente.

Resultados. En el grupo expuesto no se encontró correlación entre las concentraciones de $\mathrm{COHb}$-S y de CO-aire. La COHb-S media del grupo expuesto (2,9\%) fue significativamente superior a la del grupo de control $(1,6 \%)$ y a la concentración permisible según el método analítico empleado $(<1,5 \%)$. Todos los sintomas de frecuencia diaria afectaron a un mayor porcentaje de individuos del grupo expuesto que del grupo de control; entre los de frecuencia diaria, los más comunes en el grupo expuesto fueron la cefalea y la fatiga. En siete quioscos $(43,7 \%)$ se excedieron los limites permisibles de CO-aire.

Conclusiones. Aunque la $\mathrm{COHb}-\mathrm{S}$ media no parece alarmante, las exposiciones al $\mathrm{CO}$ registradas podrían tener un impacto adverso sobre la salud de estos trabajadores, especialmente si padecieran una alteración cardiovascular o por el resultado de la acción potenciadora con otros contaminantes ambientales. Se recomienda la realización de nuevos estudios con mediciones más frecuentes y prolongadas, para ejercer medidas de prevención y control de efectos adversos para la salud y estimar la calidad del aire.

Palabras clave Monóxido de carbono, exposición laboral, carboxihemoglobina, efectos en la salud.

Gran parte de la emisión de monóxido de carbono (CO) tiene su origen en el empleo de la gasolina como com-

\footnotetext{
Centro de Investigaciones Toxicológicas de la Universidad de Carabobo (CITUC), Valencia, Venezuela. Toda la correspondencia debe ser enviada a la siguiente dirección: CITUC. Calle 144 No. RIO211, Valencia, Venezuela. Dirección electrónica: martini@telcel. net.ve

2 Unidad de Toxicología Clínica, Facultad de Medicina, Universidad de Valladolid, Valladolid, España.
}

bustible de los vehículos a motor, especialmente en el área urbana. Otras fuentes son la generación de calor y energía, algunos procesos industriales y la incineración de desechos (1). Venezuela se encuentra entre los países latinoamericanos que han promulgado alguna legislación para el control de los contaminantes del aire, entre ellos el CO. Las concentraciones máximas permisibles establecidas en nuestro país para este gas son, $10 \mu \mathrm{g} / \mathrm{m}^{3}$ en 8 horas y $40 \mu \mathrm{g} / \mathrm{m}^{3}$ en 1 hora (2), frente a los 10 y $30 \mu \mathrm{g} / \mathrm{m}^{3}$, respectivamente, que establece la Organización Mundial de la Salud (OMS) (3). Las concentraciones de CO siempre son más altas en lugares como las avenidas de gran tránsito, los estacionamientos subterráneos o las terminales de pasajeros, y representan una exposición laboral para trabajadores como los fiscales de tránsito, 
vendedores ambulantes y de puestos fijos, policías, etc. (4).

El CO se absorbe por vía respiratoria y es rápidamente transportado en la sangre, donde se combina con la hemoglobina $(\mathrm{Hb})$ para formar carboxihemoglobina $(\mathrm{COHb})$, que tiene menor capacidad transportadora de oxígeno $\left(\mathrm{O}_{2}\right)$, por lo que puede producir hipoxia tisular (5-7).

Dependiendo de la intensidad de la exposición, los efectos de la intoxicación por $\mathrm{CO}$ pueden variar desde la cefalea hasta la muerte (8). El efecto cardíaco de bajas concentraciones de CO está bien documentado (8-12). Las diversas manifestaciones neurológicas de la exposición al CO también están bien establecidas $(9,13)$.

Los efectos de la exposición al CO están bien estudiados (8-13), pero la información referente a sus potenciales efectos en vendedores ambulantes o de puestos fijos en áreas de intenso tránsito de vehículos es extremadamente limitada. La magnitud del problema de las intoxicaciones agudas o crónicas por $\mathrm{CO}$ es prácticamente desconocida en nuestro país, ya que los casos no son regularmente registrados, excepto los más graves, que requieren hospitalización o fallecen. Valencia, ubicada en la región central de Venezuela, es la primera ciudad industrial del país, con el mayor parque empresarial y una población estimada de 1,5 millones de habitantes (14), y ocupa el tercer lugar en el número de vehículos de transporte. El propósito de esta investigación fue determinar si los vendedores que regentan puestos de venta fijos (quioscos) en el casco Norte de Valencia (un área con alta densidad de tránsito), están expuestos a cantidades no permisibles de CO, los factores concomitantes que contribuyen a la intensidad de esa exposición y sus potenciales efectos adversos para la salud.

\section{MATERIALES Y MÉTODOS}

Se realizó un estudio transversal, descriptivo, de carácter exploratorio, en el que participaron dos grupos de personas: un grupo expuesto (GE), constituido por personas que trabajan como vendedores de quioscos (pequeños establecimientos fijos de la "economía informal" que tienen una dimensión aproximada de 2,40 $\mathrm{m}^{2}$ donde se venden diferentes mercancías) ubicados en una extensión de 10 $\mathrm{km}$ en el área Norte de la avenida principal de la ciudad de Valencia, y un grupo de control (GC) constituido por docentes, estudiantes y obreros de la Universidad Simón Rodríguez en la localidad de Canoabo, ubicada a 120 $\mathrm{km}$ de Valencia, en una zona montañosa, apartada, sin contaminación ambiental aparente.

De ambos grupos se excluyeron los fumadores, ya que el hábito tabáquico puede producir concentraciones de $\mathrm{COHb}$ endógena en sangre (COHb-S), de 1,5 a $3 \%$ o más (15-17), y las embarazadas, ya que la producción endógena de CO aumenta hasta 15 partes por millón (ppm) durante el embarazo. Para el GC se exigió además que residieran en Canoabo, para evitar el contacto con contaminantes atmosféricos propios de medios de transporte y de la ciudad.

Inicialmente se elaboró un mapa de la zona estudiada en el que se señalaron los quioscos, paradas de autobuses, gasolineras, semáforos e intersecciones importantes. Después se realizó una "encuesta piloto" en la que se indujo a las personas a que participaran voluntariamente en la investigación. Esto permitió asegurar que se cumplían los criterios de exclusión y determinar las características de la muestra. La encuesta identificó un total de 34 quioscos con 48 trabajadores ( 30 hombres y 18 mujeres) de 18 a 67 años de edad. A los 20 individuos expuestos (16 quioscos) que cumplían los criterios de inclusión y aceptaron participar se les realizó una entrevista destinada a conocer sus antecedentes personales y laborales, historia clínica (con énfasis en los signos y síntomas relacionados con el $\mathrm{CO}$ ), estilos de vida (pasatiempos, consumo de alcohol), consumo de medicamentos y antigüedad en el trabajo actual $(<1-5$ años, 6-10 años o > 10 años). Se investigó la presencia y frecuencia (diaria, 4-6 veces/semana, $0-3$ veces/semana, 3-4 veces/mes, $0-2$ veces/mes o nunca) durante los últimos 6 meses de signos y síntomas que pudiesen estar relacionados con la exposición al CO. Para el GC se seleccionaron 23 individuos apareados por edades y sexo con el GE. El estudio se realizó en el último trimestre del año 1999.

En el interior de los 16 quioscos participantes se procedió al monitoreo continuo del $\mathrm{CO}$ ambiental durante 7 horas al día a lo largo de 5 días de una misma semana. Se utilizó un monitor de gases Bacharach de celdas electroquímicas equipado con un sensor de oxígeno y combustible. El monitor de $\mathrm{CO}$ fue pre-calibrado usando $50 \mathrm{ppm}$ de CO. Durante la misma semana en la que se monitorizó el CO ambiental, a cada individuo de ambos grupos se le tomó una muestra de $5 \mathrm{~cm}^{3}$ de sangre venosa en tubos heparinizados, debidamente sellados y almacenados para su envío al día siguiente al Laboratorio de la Unidad de Toxicología Clínica de la Facultad de Medicina de la Universidad de Valladolid, España, donde se determinaron las concentraciones de $\mathrm{COHb}-\mathrm{S}$ con un cooxímetro $270 \mathrm{Ciba}$ Corning. El método tiene un intervalo de referencia en la población normal de 0,0 a $1,5 \%$ de $\mathrm{COHb}$, comparado con el límite biológico de exposición de la American Conference of Governmental Industrial Hygienists (ACGHI), que es de $3,5 \%$ al final de la jornada laboral, y el del National Institute for Occupational Safety and Health (NIOSH), que es de $5 \%(18,19)$.

El análisis estadístico se realizó con el programa Statistical Package for Social Sciences (SPSS) versión 9.0. Se calcularon las frecuencias absolutas y relativas, medidas de tendencia central y de asociación (correlación de Pearson) y la significación estadística ( $t$ de Student).

\section{RESULTADOS}

Las características de la población estudiada se describen en el cuadro 1. 
CUADRO 1. Características de los grupos. Valencia, Venezuela, 1999.

\begin{tabular}{lcc}
\hline & $\begin{array}{c}\text { Grupo } \\
\text { expuesto }\end{array}$ & $\begin{array}{c}\text { Grupo de } \\
\text { control }\end{array}$ \\
\hline Sexo $^{\mathrm{a}}$ & & \\
Masculino & $13(65)$ & $8(35)$ \\
Femenino & $7(35)$ & $15(65)$ \\
Edad $^{\mathrm{b}}$ & $37,2 \pm 11,22$ & $34,5 \pm 4,62$ \\
& $(20-56)$ & $(19-60)$ \\
\hline
\end{tabular}

a Número (\%).

b Media \pm desviación estándar (intervalo).

Para los individuos del GE, la antigüedad media en el trabajo fue de 5,14 años (0,02 a 18). La mayoría (65\%) se incluyó en el grupo de $<1-5$ años de antigüedad, en el que la $\mathrm{COHb}-\mathrm{S}$ media fue de 3,2\%, seguido del grupo de 6-10 años de antigüedad (30\%), con una $\mathrm{COHb}-\mathrm{S}$ media de $2,2 \%$. No se encontró relación entre la antigüedad en el trabajo y la $\mathrm{COHb}-\mathrm{S}$ media. La concentración media ( \pm desviación estándar) de $\mathrm{COHb}$-S fue de 2,9 $\pm 1,4 \%$ (intervalo: 1,4 a $8,5 \%$ ) en el GE y de $1,6 \pm 0,6 \%$ (intervalo: 0,5 a $3,4 \%$ ) en el GC; la diferencia fue estadísticamente significativa $(t=3,90 ; p<0,001)$. En el GE, la concentración media de CO-aire, de 23,8 $\pm 4,1$ ppm (intervalo: 14,8 a 34,1 ppm), no se correlacionó significativamente con la concentración media de $\mathrm{COHb}-\mathrm{S}$ (coeficiente de correlación de Pearson, $r=0,087)$. Tanto los valores de $\mathrm{COHb}$ $\mathrm{S}$ como los de $\mathrm{CO}$-aire siguieron una distribución normal en este estudio. En siete quioscos $(43,7 \%)$ se excedieron los límites permisibles de $\mathrm{CO}$-aire.

Como era de esperar, cuando se comparó la $\mathrm{COHb}-\mathrm{S}$ media de ambos grupos con el valor de referencia según el método del cooxímetro $(<1,5 \%)$, la concentración fue significativamente mayor en el GE ( $t=4,195 ; p=0,001)$, pero no en el GC ( $t=0,428 ; p=0,673)$. Tanto en el GE como en el GC, la COHb-S media fue inferior al límite biológico de exposición recomendado por la ACGIH
$(3,5 \%)$ y la diferencia fue significativa en el GC. La diferencia entre la concentración media de CO-aire y las concentraciones permisibles según la ACGIH (18) no fue significativa.

El cuadro 2 describe los signos y síntomas que en el GE tuvieron una frecuencia $>15 \%$ y el cuadro 3 relaciona los síntomas más frecuentes en los dos grupos (frecuencia diaria o de 4-6 veces/semana) con las concentraciones de $\mathrm{COHb}-\mathrm{S}$. Cabe destacar que todos los síntomas de frecuencia tanto diaria como de 4-6 veces/semana afectaron a un mayor porcentaje de indivi-

CUADRO 2. Distribución en ambos grupos de los signos y síntomas con frecuencia $>15 \%$ en el grupo expuesto. Valencia, Venezuela, 1999

\begin{tabular}{lccccr}
\hline & \multicolumn{2}{c}{ Grupo expuesto } & & \multicolumn{2}{c}{ Grupo de control } \\
\cline { 2 - 3 } Signos y síntomas & No. & $\%^{\mathrm{a}}$ & & No. & $\%^{\mathrm{a}}$ \\
\hline Dificultad de audición & 3 & 15 & & 1 & 4 \\
Disnea & 3 & 15 & & 3 & 13 \\
Dolor abdominal & 3 & 15 & & 2 & 9 \\
Flujo nasal & 3 & 15 & & 2 & 9 \\
Tos & 3 & 15 & & 2 & 9 \\
Problemas visuales & 5 & 25 & & 5 & 22 \\
Somnolencia & 7 & 35 & & 7 & 30 \\
Cefalea & 8 & 40 & & 12 & 52 \\
Irritación nasal & 8 & 40 & & 4 & 17 \\
Mareo & 8 & 40 & & 4 & 17 \\
Irritación ocular & 12 & 60 & & 5 & 22 \\
Fatiga & 13 & 65 & 7 & 30 \\
a Porcentajo calculado & 5 & & & & \\
\hline
\end{tabular}

a Porcentaje calculado sobre el total de individuos del grupo.

CUADRO 3. Distribución de los signos y síntomas de frecuencia "diaria" y "4-6 veces/semana" en los grupos expuesto y de control y su relación con la concentración media de carboxihemoglobina en sangre (COHb-S). Valencia, Venezuela, 1999

\begin{tabular}{|c|c|c|c|c|c|c|c|c|c|c|c|c|}
\hline \multirow{3}{*}{ Signos/síntomas } & \multicolumn{12}{|c|}{ Frecuencia } \\
\hline & \multicolumn{6}{|c|}{ Diaria } & \multicolumn{6}{|c|}{ 4-6 veces/semana } \\
\hline & \multicolumn{3}{|c|}{ Grupo expuesto } & \multicolumn{3}{|c|}{ Grupo de control } & \multicolumn{3}{|c|}{ Grupo expuesto } & \multicolumn{3}{|c|}{ Grupo de control } \\
\hline Cefalea & 5 & 25 & 3,74 & 1 & 4 & 1,40 & 2 & 10 & 2,80 & 2 & 9 & 1,30 \\
\hline Fatiga & 5 & 25 & 3,60 & 1 & 4 & 2,00 & 4 & 20 & 2,35 & 2 & 9 & 1,05 \\
\hline Mareo & 0 & - & - & 0 & - & - & 2 & 10 & 2,40 & 1 & 4 & 3,40 \\
\hline Irritación ocular & 4 & 20 & 2,52 & 1 & 4 & 0,90 & 0 & - & - & 0 & - & - \\
\hline Problemas visuales & 4 & 20 & 4,07 & 3 & 13 & 2,16 & 0 & - & - & 0 & - & - \\
\hline Somnolencia & 3 & 15 & 2,40 & 2 & 9 & 1,55 & 0 & - & - & 0 & - & - \\
\hline Tos & 2 & 10 & 2,55 & 1 & 4 & 2,00 & 0 & - & - & 0 & - & - \\
\hline
\end{tabular}


CUADRO 4. Concentraciones medias de carboxihemoglobina en la sangre (COHb-S) $y$ de monóxido de carbono en el aire (CO-aire) en el grupo expuesto, según la ubicación de los quioscos. Valencia, Venezuela, 1999

\begin{tabular}{lcrcc}
\hline Sitio crítico & $\mathrm{F}$ & $\%$ & $\begin{array}{c}\text { COHb-S } \\
(\%)\end{array}$ & $\begin{array}{c}\text { CO-aire } \\
(\mathrm{ppm})\end{array}$ \\
\hline PA & 2 & 10 & 3,05 & 20,15 \\
EPA & 5 & 25 & 3,94 & 23,54 \\
EG & 1 & 5 & 2,00 & 26,10 \\
II & 1 & 5 & 2,40 & 26,80 \\
PA/EG & 3 & 15 & 2,10 & 24,43 \\
II/PAO/S & 3 & 15 & 2,66 & 27,30 \\
EPA/EG & 1 & 5 & 3,20 & 23,20 \\
NADA & 4 & 20 & 2,35 & 21,90 \\
TOTAL & $20^{\text {a }}$ & 100 & 2,85 & 23,84 \\
\hline
\end{tabular}

PA: Parada de autobús; EPA: enfrente de una parada de autobús; PAO: parada de autobús en acera opuesta; EG: estación de gasolina; S: semáforo; II: intersección importante. a 20 personas en 16 quioscos.

duos del GE que del GC. La diferencia entre las concentraciones de $\mathrm{COHb}-\mathrm{S}$ en el GE y el GC solo fue significativa para los pacientes con cefalea 4-6 veces/semana $(t=10,60 ; p=0,03)$.

El cuadro 4 muestra las concentraciones medias de $\mathrm{COHb}-\mathrm{S}$ y $\mathrm{CO}$-aire en función de la ubicación de los quioscos. La mayor concentración de CO-aire (27,3 ppm) correspondió a los quioscos ubicados junto a tres sitios críticos (II/PAO/S), y la mayor concentración de $\mathrm{COHb}-\mathrm{S}(3,94 \%)$ a los trabajadores cuyos quioscos se encontraban enfrente de una parada de autobús (EPA). Sin embargo, solo se encontró una correlación positiva en el GE, en cuanto al sitio crítico PA, es decir, que en la medida que aumentan los niveles de $\mathrm{CO}$-aire en esos quioscos, pueden aumentar los niveles de $\mathrm{COHb}-\mathrm{S}$ en los sujetos que allí trabajan.

\section{DISCUSIÓN}

Las concentraciones de $\mathrm{COHb}-\mathrm{S}$ registradas en este estudio son superiores a las descritas por otros investigadores, como Soto et al. (20), quienes encontraron concentraciones de 1,6\% en personas que trabajan en zonas con- taminadas de Puebla, México, y de $0,43 \%$ en los que habitan en zonas rurales, no contaminadas. Por otra parte, aunque solo se superaron los valores permisibles de CO-aire en el 43,7\% de los quioscos, parece que no existe una concentración de $\mathrm{CO}$ que no ejerza un estrés significativo en pacientes con enfermedad cardiovascular.

Los síntomas neurológicos observados son sutiles, inespecíficos y difíciles de detectar en un examen neurológico habitual (21). Los más comunes entre los de frecuencia diaria (cuadro 3) fueron la cefalea y la fatiga (25\% cada uno), seguidos por la irritación nasal y ocular y los problemas visuales $(20 \%$ cada uno). La cefalea y la fatiga son síntomas tempranos de neurotoxicidad que pueden ocurrir con bajos grados de exposición que producen concentraciones de $\mathrm{COHb}-\mathrm{S}$ de 0,2 a 0,3\% (22).

La fatiga se asoció con la tercera cifra más elevada de $\mathrm{COHb}-\mathrm{S}$, tras los problemas visuales y la cefalea. Su aparición se podría explicar porque al aumentar la $\mathrm{COHb}-\mathrm{S}$ disminuye el umbral de la acidosis metabólica, con lo que el metabolismo anaeróbico puede aparecer más temprano, causando fatiga al músculo esquelético (12). Sin embargo, no se puede descartar que otros factores distintos de la contaminación ambiental, tales como el ruido, el estrés y la incomodidad física causados por el tráfico en la zona estudiada, también estén implicados en la producción de estos efectos. En cuanto a los problemas visuales, Amitai et al. (23) han descrito alteraciones funcionales neurológicas y disminución de la visión asociados con niveles de $\mathrm{COHb}-\mathrm{S}$ de $0,03-0,05 \%$.

En el GE no se describieron signos y síntomas cardiovasculares como bradicardia, alteraciones electrocardiográficas, angina de pecho, infarto de miocardio y arritmias cardíacas; los individuos estudiados solo manifestaron taquicardia, con una frecuencia de $5 \%$ tanto en el GE como en el GC. Esto puede deberse a que el $\mathrm{CO}$ exacerba síntomas como la angina durante el ejercicio con concentraciones de $\mathrm{COHb}-\mathrm{S}$ de $2,9-4,5 \%(24,25)$, que no se alcanzaron en este estudio. Sin embargo, en presencia de trastornos cardiovasculares agravantes se ha observado una disminución de la capacidad para el trabajo en condiciones de ejercicio máximas con niveles de $\mathrm{COHb}-\mathrm{S}$ de $2,5 \%$ y aun menores (26).

Los resultados de este estudio indican que, aún cuando no se sobrepasen los límites permisibles establecidos, la actividad laboral de los individuos estudiados representa un riesgo potencial para su salud debido a la exposición al CO, pues el 100\% de los síntomas con frecuencia diaria o de 4-6 veces/semana ocurrieron en un mayor porcentaje de individuos del GE que del GC, menos expuestos a los contaminantes atmosféricos.

En el $43,7 \%$ de los quioscos se superaron las concentraciones permisibles de $\mathrm{CO}$-aire. Aunque las cifras de $\mathrm{COHb}-\mathrm{S}$ y CO-aire encontradas no eran alarmantes, tales exposiciones podrían tener efectos adversos sobre la salud debido a la coexistencia de trastornos cardiovasculares o a la interacción aditiva con otros contaminantes químicos ambientales, ruido, temperaturas elevadas, etc., en sus puestos de trabajo. Aunque es posible que los transeuntes de la zona también se vean afectados, el presente estudio no permite estimar esos efectos.

En resumen, la concentración media de $\mathrm{COHb}-\mathrm{S}$ fue significativamente mayor en el GE que en el GC, y también significativamente mayor que el límite permisible según el método analítico empleado. El área estudiada es una zona crítica que debe ser objeto de vigilancia de la calidad del aire.

En general en Venezuela, no disponemos de datos epidemiológicos adecuados para evaluar de manera sistemática y rigurosa los efectos que padecen las poblaciones expuestas diariamente a los contaminantes que se vierten a la atmósfera en áreas como la estudiada. Los registros actuales de morbilidad en nuestro país no permiten establecer relaciones causales entre las enfermedades y los contaminantes del ambiente en general, y 
mucho menos con los diferentes tipos de contaminantes.

Con el fin de intentar establecer una relación causa-efecto y estimar mejor la magnitud del problema, se recomienda la realización de estudios sistemáticos más prolongados, orientados hacia los efectos de las concentraciones relativa- mente bajas de CO-aire, y la correspondiente $\mathrm{COHb}-\mathrm{S}$, sobre funciones como la conducción de vehículos, la percepción en otras tareas y las enfermedades más probablemente relacionadas con la contaminación por este gas.

La divulgación de estos resultados tiene evidente relevancia regional, ya que la medición efectuada constituye un buen indicador de las condiciones existentes en la ciudad de Valencia y permitiría, si se dispusiera de recursos para estudios posteriores, estudiar las tendencias del problema y extrapolar estos resultados a zonas similares regionales o nacionales.

\section{REFERENCIAS}

1. Organización Panamericana de la Salud/ Organización Mundial de la Salud. Criterios de salud ambiental 13. Monóxido de carbono. Washington: OPS/OMS; 1983. p 4.

2. Gaceta Oficial de la República de Venezuela, No. 4899, Según decreto No. 638, Caracas 19-5-1995

3. Weitzenfeld H. Contaminación atmosférica y salud en América Latina. Bol Oficina Sanit Panam 1992; 112:98-109.

4. Gutiérrez JH. Exposición humana a los contaminantes del aire. En: Gutiérrez H, Corey G y Romieu I, eds. Contaminación del aire y riesgos para la salud. México ECO/OPS/OMS, 1991. pp. $99-100$

5. Guyton AC. Human physiology and mechanisms of disease, 5 th ed. Toronto: W. B. Saunders Company; 1992.

6. Raub JA, Mathieu-Nolf M, Hampson NB, Thom SR. Carbon monoxide poisoning: a public health perspective. Toxicology 2000; 145: 1-14.

7. Manahan SE. Environmental chemistry. Chapter 18: Environmental biochemistry and chemical toxicology. 6a ed. Boca Raton: Lewis; 1994

8. McCammon JB, McKenzie LE, Heinzman M. Carbon monoxide poisoning related to the indoor use of propane-fueled forklifts in Colorado workplaces. Appl Occup Environ Hyg 1996;11:192-198.

9. Ellenhorn MJ. Carbon monoxide. En: Ellenhorn's medical toxicology: diagnosis and treatment of human poisoning. 2nd ed. Baltimore: Williams \& Wilkins; 1997. pp. 14651476.

10. Koskela RS. Cardiovascular diseases among foundry workers exposed to carbon monoxide. Scand J Work Environ Health 1994;20: 286-293.
11. Sheps DS, Herbst MC, Hinderliter AL, Adams KF, Ekelund LG, O'Neil JJ, et al. Production of arrhythmias by elevated carboxyhemoglobin in patients with coronary artery disease. Ann Intern Med 1990;113:343-351.

12. Adir Y, Merdler A, Ben Haim S, Front A, Hardruf $\mathrm{R}$, Bitterman $\mathrm{H}$. Effects of exposure to low concentrations of carbon monoxide on exercise performance and myocardial perfusion in young healthy men. Occup Environ Med 1999;56:535-538.

13. Heckerling PS, Leikin JB, Terzian CG, Maturen A. Occult carbon monoxide poisoning in patients with neurologic illness. J Toxicol Clin Toxicol 1990;28:29-44.

14. Feo CO. Dirección de planificación. Secretaría Estatal de Planificación, Ambiente y Ordenación del Territorio (DEPLAN). Estadísticas de Estado Carabobo, Marzo 1997.

15. Burnett RT, Cakmak SR, Raizenne ME, Stieb D, Vincent R, Krewsky D, et al. The association between ambient carbon monoxide levels and daily mortality in Toronto, Canada. J Air Waste Manag Assoc 1998;48:689-700.

16. Hewat VN, Foster EV, O'Brien GD, Town GI Ambient and exhaled carbon monoxide levels in a high traffic density area in Christchurch N Z Med J 1998;111:343-344.

17. Wald N, Idle M, Boreham J, Bailey A. Carbon monoxide in breath in relation to smoking and carboxyhaemoglobin levels. Thorax 1981; 36:366-369.

18. American Conference of Governmental Industrial Hygienists. Threshold limit values for chemical substances and physical agents and biological exposure indices. 1998-1999. Cincinnati, OH: ACGIH; 1999.

19. Woebkenberg ML. Direct-reading monitoring devices for carbon monoxide. Appl Occup Environ Hyg 1998;13:567-569.
20. Soto Rojas G, Márquez Cabrera T, Adams R. Niveles de carboxihemoglobina en la población de Puebla y sus implicaciones. Salud Publica Mex 1981;23:399-403.

21. Seger D, Welch L. Carbon monoxide controversies: neuropsychological testing, mechanism of toxicity and hyperbaric oxygen. Ann Emerg Med 1994;24:242-248.

22. Thom SR, Keim LW. Carbon monoxide poisoning: a review. Epidemiology, pathophysiology, clinical findings and treatment options including hyperbaric oxygen therapy. Clin Tox 1989;27:141-156.

23. Amitai Y, Zlotogorski Z, Golan-Katzav V, Vexler A y Gross D: Neuropsychological impairment from acute low-level exposure to carbon monoxide. Arch Neurol 1998;55: 845-848.

24. Allred EN, Bleeker ER, Chaitman BR, Dahms TE, Gottieb SO, Hackney JD, et al. Short-term effects of carbon monoxide exposure on the exercise performance of subjects with coronary artery disease. N Engl J Med 1989;321: 1426-1432.

25. Allred EN, Bleeker ER, Chaitman BR, Dahms TD, Gottieb SO, Hackney JD, et al. Effects of carbon monoxide on myocardial ischemia Environ Health Perspect 1991;91:89-102.

26. Haflidson WO, Figueira CS: Carbon monoxide exposure of concrete finishers. Appl Occup Environ Hyg 1998;12:126-131.

Manuscrito recibido el 13 de julio de 2000. Aceptado para publicación, tras revisión, el 9 de marzo de 2001. 
ABSTRACT Objective. To study the level of exposure to carbon monoxide (CO), factors contributing to this exposure, and the exposure's potential effects on the health of vendors who have kiosks located on an avenue with heavy vehicular traffic in the city of Valencia, Venezuela.

\section{Evaluation of exposure to carbon monoxide among kiosk vendors in Valencia, Venezuela}

Methods. We measured the concentration of CO in the air (CO-air) in 16 kiosks in Valencia and also determined the concentration of carboxyhemoglobin $(\mathrm{COHb})$ in the blood of 20 individuals who worked in those kiosks. We also determined the $\mathrm{COHb}$ concentrations in a control group made up of 23 professors, students, and workers from Simón Rodríguez University, which is in a town some $120 \mathrm{~km}$ from Valencia, in a remote mountainous area without noticeable pollution.

Results. In seven of the Valencia kiosks (43.7\%) the permissible CO-air limits were exceeded. Among the vendors experimental group there was no correlation between the concentrations of $\mathrm{CO}$-air and of $\mathrm{COHb}$. The $\mathrm{COHb}$ average for the experimental group $(2.9 \%)$ was significantly higher than that of the control group $(1.6 \%)$ and that of the permissible level, according to the analytical method used $(<1.5 \%)$. Of a group of symptoms possibly related to $\mathrm{CO}$ exposure that were assessed as to whether or not they occurred on a daily basis, all of these symptoms affected a larger percentage of individuals in the experimental group than in the control group. The most common of these symptoms among the experimental group were fatigue and strong headaches.

Conclusions. While the vendors' $\mathrm{COHb}$ average does not seem alarming, the $\mathrm{CO}$ exposures found could have an adverse impact on these workers' health, especially if they suffered from cardiovascular disorders, or as the result of additive interactions with other environmental pollutants. We recommend carrying out additional studies with more frequent and extended measurements of air quality, to produce information that could be applied in efforts to prevent and control adverse $\mathrm{CO}$ health effects.

Séptimo Curso Internacional: $\boxminus$ dengue, un desafío en el tercer milenio

$\begin{array}{ll}\text { Fecha: } & 13 \text { al } 24 \text { de agosto de } 2001 \\ \text { Lugar: } & \text { Gudad Habana, Ouba }\end{array}$

日 Centro Colaborador de la OPS/OMS para el Estudio de las Enfermedades Víricas y el Centro Colaborador de la OPS/OMS para el Adiestramiento en Investigaciones en Malacología Médica y Control Biológico de Vectores y Huéspedes Intermediarios del Instituto de Medicina Tropical "Pedro Kourí", La Habana, Ouba, con el auspicio de la Organización Panamericana de la Salud (OPS) y del Programa Especial para Investigación y Entrenamiento en Enfermedades Tropicales de la Organización Mundial de la Salud (TDR/OMS), realizará en Gudad Habana, Cuba, del 13 al 24 de agosto del 2001, este séptimo curso internacional sobre el dengue, de carácter multidisciplinario, con sesiones teóricas y prácticas, en el que se analizarán los conocimientos más novedosos sobre el tema.

日 curso está dirigido a médicos, microbiólogos, infectólogos, bioquímicos, epidemiólogos, entomólogos y técnicos relacionados con el temas del dengue y su control. Su costo es de US\$1000 (matrícula, material docente, cóctel de bienvenida y cena de despedida). Todas las presentaciones serán hechas en español.

Las solicitudes de matrícula deben dirigirse a:

Prof. María G Guzmán, Instituto "Pedro Kourí"

Autopista Novia del Mediodía, km 6, Apartado Postal Mnao 13. 13, Gudad Habana, Cuba. Teléfono: 53-7-220450; 53-7-220633. Fax: 53-7-220633; 53-7-246051

Más información en: http://infonew.sld.cu/instituciones/ipk/cdengue.htm 